Mens

Revue d'histoire intellectuelle et culturelle

mens

\title{
L'Inde et la décolonisation au Canada français
}

\section{Serge Granger}

Volume 13, numéro 1, automne 2012

S’approprier le passé des autres : les usages de l'histoire internationale au Québec avant la Révolution tranquille

URI : https://id.erudit.org/iderudit/1019698ar

DOI : https://doi.org/10.7202/1019698ar

Aller au sommaire du numéro

Éditeur(s)

Centre de recherche en civilisation canadienne-française

ISSN

1492-8647 (imprimé)

1927-9299 (numérique)

Découvrir la revue

Citer cet article

Granger, S. (2012). L’Inde et la décolonisation au Canada français. Mens, 13(1), 55-79. https://doi.org/10.7202/1019698ar

\section{Résumé de l'article}

L'indépendance de l'Inde a contribué à diffuser un discours sur la décolonisation dans le monde entier, notamment au Canada français. Exemple d'une colonie qui se libère de l'Empire britannique - symbole de l'aliénation coloniale -, l'Inde incarne une idée de la décolonisation qui sera adoptée par les Canadiens français dans la mesure où elle leur fournit les outils conceptuels pour comprendre leur condition historique et la situer à l'échelle mondiale. À l'aide de périodiques québécois, dont Le Devoir et L'Action nationale, cet article retrace les références à l'indépendance de l'Inde et examine comment elle nourrit le discours sur la décolonisation au Canada français avant la Révolution tranquille. 


\title{
L'Inde et la décolonisation au Canada français
}

\author{
Serge Granger \\ École de politique appliquée \\ Université de Sherbrooke
}

\section{Résumé}

L'indépendance de l'Inde a contribué à diffuser un discours sur la décolonisation dans le monde entier, notamment au Canada français. Exemple d'une colonie qui se libère de l'Empire britannique - symbole de l'aliénation coloniale -, l'Inde incarne une idée de la décolonisation qui sera adoptée par les Canadiens français dans la mesure où elle leur fournit les outils conceptuels pour comprendre leur condition historique et la situer à l'échelle mondiale. À l'aide de périodiques québécois, dont Le Devoir et L'Action nationale, cet article retrace les références à l'indépendance de l'Inde et examine comment elle nourrit le discours sur la décolonisation au Canada français avant la Révolution tranquille.

\section{Abstract}

Indian independence played a significant role in the transmission of the discourse on decolonization around the world, notably in French Canada. India offers not only the example of a British colony freeing itself from the British Empire, the symbol of colonial alienation, but it becomes the incarnation of a certain idea of decolonization that will be adopted in French Canada. This discourse offers to French Canadians a globalized conception of their historical condition. Through the use of Québécois journals and newspapers, notable Le Devoir and L'Action nationale, this article analyses references to Indian independence and show how it 
shapes a French-Canadian discourse on decolonization well before the Quiet Revolution.

Pour plusieurs nationalistes canadiens-français, leur lutte politique est comparable à celle d'autres peuples colonisés de la terre qui revendiquent leur autodétermination. Bien qu'il soit exagéré de considérer l'indépendance de l'Inde comme un facteur ayant déclenché la mutation du discours nationaliste au Canada français, elle a néanmoins aidé à diffuser un modèle conceptuel de la décolonisation souvent exploité par les nationalistes canadiens-français et québécois dans les années 1960. Ainsi, l'espace politique de l'Empire britannique a permis aux nationalistes canadiens-français et indiens de partager leur condition de subalternes. La prise de conscience de cet état d'infériorité à l'origine des revendications du tiers-monde, dont celles de l'Inde, participe au changement du paradigme nationaliste qui construit une hybridité nouvelle dans un Québec qui s'identifie alors davantage aux luttes de décolonisation du Sud.

Linfluence de l'indépendance de l'Inde sur la prise de conscience de la domination à travers le monde est réelle : non seulement le pays organise-t-il les grandes conférences afro-asiatiques de la décolonisation, mais en plus il favorise la formation et la diffusion d'un discours politique anticolonialiste qui atteint son paroxysme au début des années 1960, l'ONU adoptant même, en 1961, une résolution abolissant le colonialisme ${ }^{1}$. Mark Twain n'avait-il pas qualifié l'Inde de mère de l'histoire? On peut certainement affirmer que son expérience historique annonce l'émancipation des peuples du monde. La construction du discours sur la décolonisation pousse l'Inde à mener le mouvement des non-alignés qui conduit à l'affirmation politique des pays du Sud. Indéniablement, le monde indien agit comme

\footnotetext{
${ }^{1}$ Ce positionnement n'est pas étranger à l'émergence subséquente des études subalternes comme discipline universitaire; celles-ci ont débuté en 1982 à l'Université de Delhi autour de Ranajit Guha, qui publie les six premiers numéros des Subaltern Studies.
} 
puissance globalisante par son économie, sa diaspora, sa culture et, bien sûr, son discours politique, même au Québec.

Dans un rapport politique secret (aujourd'hui déclassifié), Hardit Singh Malik, haut-commissaire de l'Inde au Canada, compare les perceptions des Canadiens anglais et des Canadiens français au sujet de l'indépendance de son pays en 1947. Il souligne que le Canada anglais, qui considère l'attachement de l'Inde au Commonwealth comme l'adhésion à une famille politique, la perçoit amicalement, tandis que les Canadiens français s'en réjouissent, la considérant comme un symbole de liberté2.

Je me propose donc de vérifier cette observation de Malik en analysant les périodiques canadiens-français qui s'intéressaient à l'Inde à l'époque afin de savoir si son indépendance est effectivement perçue comme un symbole de liberté. La plupart des documents examinés sont des quotidiens francophones publiés entre 1937 et 1948. Des moments charnières ont été ciblés, comme le déclenchement de la guerre en 1939, l'attaque de Pearl Harbor et de Hong Kong par le Japon en décembre 1941, le lancement du mouvement Quit India de Gandhi en août 1942, la libération des leaders du Congrès indien en 1944, la capitulation du Japon en août 1945 et l'élection générale de janvier 1946. Les mois de juillet et d'août 1947, qui voient l'aboutissement du mouvement pour l'indépendance, ont été examinés en entier dans les quotidiens suivants : Le Devoir, La Presse, Le Soleil, La Tribune, Le Canada, La Patrie et L'Action catholique. Seuls les articles écrits par des éditorialistes ou des journalistes ont été retenus. Dans certains cas, dont ceux de La Presse et de La Tribune, les dépêches des agences de presse internationales furent nombreuses, occupant parfois plusieurs pages, notamment lors de l'indépendance et de l'assassinat de Gandhi, mais seuls les commentaires des Canadiens français ont été considérés. Des périodiques influents susceptibles de s'intéresser à l'Inde, comme L'Action nationale, ont été dépouillés

\footnotetext{
${ }^{2}$ National Archives of India, Government of India, Ministry of External Affairs, file F.20/47-OSIII, 1947, Political Summary October 1947, no. HCC/R-1/47.
} 
dans le but de montrer que l'appropriation du concept de décolonisation par les Canadiens français précède les écrits produits au cours de la Révolution tranquille.

\section{La décolonisation avant la Révolution tranquille}

La littérature postcoloniale au Québec attribue aux ouvrages de Frantz Fanon et d'Albert Memmi une grande influence chez les intellectuels québécois de la Révolution tranquille, mais peu d'auteurs considèrent que l'indépendance de l'Inde ait pu servir de modèle ayant influé sur le développement du discours décolonisateur au Québec. Dans un article qu'il consacre aux relations indoquébécoises, Fernand Harvey affirme que « des liens historiques et contemporains, encore relativement peu connus, unissent l'Inde et le Québec ${ }^{3}$ ". Seule la revue Synergies Inde du Groupe d'études et de recherches pour le français langue internationale (GERFLINT) a préparé un numéro spécial sur les relations Inde-Québec dans le cadre des célébrations du $400^{\mathrm{e}}$ anniversaire de la ville de Québec ${ }^{4}$. Aucune monographie ne porte sur les relations du Québec avec l'Asie du Sud, sauf quelques livres écrits par des capucins relatant leurs missions en Inde. Il existe certains ouvrages qui comparent le nationalisme québécois et certains mouvements autonomistes, comme ceux du Cachemire et d'Assam, mais aucun d'entre eux n'abordent la connectivité entre le Québec et l'Indes. Plusieurs raisons peuvent expliquer la rareté des études sur le sujet : 1) peu d'auteurs indiens ont été traduits en français, ce qui limite la

${ }^{3}$ Fernand Harvey, "Le Québec et l'Inde : jalons historiques et contemporains d'une relation en construction ", dans Kichenassamy Madanagobalane (dir.), Québec : traditions, transition, transgression, Chennai, Samhita Publications, 2010, p. 100-126.

4 Serge Granger, "Les Lotbinière au Cachemire avant la Première Guerre mondiale ", Synergies Inde, $\mathrm{n}^{\circ} 3$ (2008), p. 129-140.

${ }^{5}$ Mahendra Prasad Singh et Chandra Mohan (dir.), Regionalism and National Identity: Canada India: Interdisciplinary Perspectives, Columbia, South Asia Books, 1994; Reeta Saxena, Situating Federalism: Mechanisms of Intergovernmental Relations in Canada and India, New Delhi, Manohar Books, 2006. 
production d'une indologie au Québec; 2) l'éloignement géographique et la distance culturelle entre l'Inde et le Québec freinent la possibilité d'échanges transculturels et politiques; 3) la rareté des études sur l'Inde au Québec n'encourage pas la connectivité académique entre chercheurs.

Selon l'historiographie courante, le concept de décolonisation serait apparu au Québec pendant la Révolution tranquille. En effet, au cours de cette période, les Canadiens français utilisent à profusion le terme "décolonisation ", comme l'attestent de nombreuses publications portant sur la situation "coloniale» du Québec : Le Québec est-il une colonie? (Raymond Barbeau, 1962); Le colonialisme au Québec (André D’Allemagne, 1966); Parti pris (1964). Cette revue milite pour un Québec indépendant, socialiste et laïque, trois valeurs partagées par la constitution indienne $e^{6}$. Au cours des premières années, ses publications sont consacrées à défendre l'idée que le Québec est colonisé et qu'une révolution sociale est nécessaire à sa libération. Les intellectuels Pierre Maheu, Paul Chamberland, Jean-Marc Piotte, André Major et des écrivains tels Hubert Aquin, Gaston Miron et Gérald Godin dénoncent la situation coloniale du Canada français et en proposent la renaissance par la création d'un État socialiste et indépendant ${ }^{7}$. Charles Gagnon, secrétaire général du groupe En lutte!, mentionne que les rédacteurs de Parti pris « avaient inventé le "nouveau nationalisme", supposé, comme dans les colonies, être porteur de progrès social ${ }^{8}$ ». En tant que directeur de la revue Liberté, Aquin fait connaître le Portrait du colonisé (1957) d'Albert Memmi, un auteur qu'il a rencontré lors du tournage du film $A$ l'heure de la décolonisation, produit par l'Office national du film du Canada'. Une version

\footnotetext{
${ }^{6}$ Pierre-Luc Bégin, «Parti pris : un phénomène majeur méconnu ", Québec français, $\mathrm{n}^{\circ} 153$ (printemps 2009), p. 48-50.

${ }^{7}$ Nicole Laurin, "Genèse de la sociologie marxiste au Québec ", Sociologie et sociétés, vol. 37, n 2 (automne 2005), p. 183-207.

${ }^{8}$ Charles Gagnon, «Il était une fois... Conte à l'adresse de la jeunesse de mon pays ", Bulletin d'histoire politique, vol. 13, $\mathrm{n}^{\circ} 1$ (automne 2004), p. 45.

${ }^{9}$ Guylaine Massoutre, Itinéraires d'Hubert Aquin, Montréal, Bibliothèque québécoise, 1992, p. 124.
} 
canadienne-française du Portrait du colonisé paraît aux éditions du Bas-Canada en 1963 sous les presses de Raoul Roy, éditeur de la Revue socialiste et des Cahiers de la décolonisation. À l'été 1964, Parti pris publie un numéro spécial intitulé Portrait du colonisé québécois dans lequel des textes de Chamberland ("De la damnation à la liberté ») et de Maheu ("L'CEdipe colonial ») marqueront le passage du vocable Canadien français à celui de Québécois.

Plusieurs auteurs soulignent que le traitement réservé à la Révolution tranquille en littérature est directement inspiré du discours de la décolonisation de l'après-guerre. Selon Sandra Hobbs ${ }^{10}$, la transformation de l'écriture de la résistance au Québec se produit dans les années 1950, lorsque les écrivains abandonnent le modèle de la survivance, autant dans leurs écrits que dans leur identité, et adoptent un discours au diapason des luttes coloniales du monde. Elle affirme que diverses tendances sont présentes dans la littérature de la résistance au Québec : l'une d'elles se traduit, dans le cadre d'une opposition formelle au colonisateur, par le repli et la survivance; l'autre s'exprime par mimétisme entre le colonisé et le colonisateur. Enfin, un troisième courant suit le rythme des luttes internationales. Homi Bhabha ${ }^{11}$ développera le concept d'hybridité selon lequel la résistance du colonisé peut être moderne, non traditionnelle et en mutation continuelle. Ainsi, selon Hobbs et Bhabha, le discours nationaliste de la Révolution tranquille ayant recours au concept de décolonisation serait davantage un modèle d'hybridité, car il revendique un statut identitaire moderne et international (les luttes coloniales des années 1947-1970). En prenant deux régions aussi lointaines par la géographie et la culture, mais qui sont toutes deux des colonies britanniques, on élargit les variables des études postcoloniales en postulant l'existence d'une identité périphérique commune

${ }^{10}$ Sandra Hobbs, «De l'opposition à l'ambivalence : la théorie postcoloniale et l'écriture de la résistance au Québec ", Québec Studies, n 35 (printemps-été 2003), p. 99-111.

${ }^{11}$ Homi Bhabha, Les lieux de la culture : une théorie postcoloniale, Paris, Payot, 2007. 
entre le Canada français et l'Inde au sein du discours décolonisateur. L'hybridité du discours nationaliste au Canada français emprunte à l'Inde la notion identité coloniale qui, par sa déclaration d'indépendance en 1947, introduit le postcolonialisme. Ainsi, l'Inde est à l'origine de la décolonisation dont s'inspireront d'autres peuples en s'identifiant à cette lutte et en souhaitant l'avènement de leur propre monde postcolonial. Linda Hutcheon ${ }^{12}$ précise que cette identité doit être modulée, car la variabilité des statuts des colonies limite la comparaison entre les peuples. Ainsi, comparer les Canadiens français et les Indiens peut se révéler périlleux. Pour éviter cet écueil, les études doivent relever les références à l'Inde dans le discours politique canadien-français plutôt que chercher à savoir si les Canadiens français furent des colonisés comme les Indiens. Memmi a exposé ses réticences sur le bien-fondé de qualifier le Québec d'État colonisé, ayant assez vite perçu les limites de cette comparaison. Par contre, l'appropriation par les Canadiens français du concept de décolonisation ne fait pas de doute, comme l'atteste la littérature engagée de la Révolution tranquille.

Au moment de l'indépendance de l'Inde, le nationalisme canadien-français accélère sa transformation en adoptant une approche plus moderne et plus proche de la mouvance mondiale de la décolonisation. Dès les années 1940, plusieurs événements favorisent cette transformation : Refus global, la Déclaration universelle des droits de l'homme, la création de l'ONU, la migration intensive, etc., ainsi que l'indépendance de l'Inde et la vague de décolonisation qu'elle suscite en Asie et ailleurs dans le monde. En plus de l'indépendance de l'Inde, mentionnons la guerre en Indochine et l'unification de la Chine par Mao. Jawaharlal Nehru organise la première conférence internationale du monde colonisé en 1947, quelques semaines avant de déclarer l'indépendance. Cette première dans l'histoire inter-

${ }^{12}$ Linda Hutcheon, "Circling the Downspout of Empire: Post-colonialism and Postmodernism ", ARIEL: A Review of International English Literature, vol. 20, $\mathrm{n}^{\circ} 4$ (1989), p. 149-175. 
nationale entraîne l'organisation d'autres conférences qui sonnent non seulement le réveil de l'Orient, mais aussi celui de l'Afrique et d'autres régions du monde. La deuxième conférence, tenue à New Delhi en janvier 1949, somme les Pays-Bas de quitter l'Indonésie

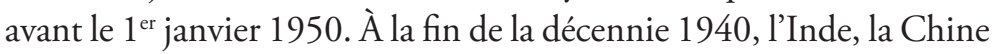
et l'Indonésie ont renversé la domination coloniale et obtenu leur indépendance. Quant au Canada français, au lendemain de la Deuxième Guerre mondiale, il connaît une campagne nationaliste qui l'encourage à se séparer de Londres, voire à devenir une république en abandonnant la monarchie constitutionnelle. Ce mouvement est un indice de la perte de puissance de l'Angleterre et de l'entrée du Canada parmi les satellites des États-Unis; de plus il contribue à la déconstruction du monde colonial.

Cette renaissance du nationalisme canadien passe par l'abandon des références à l'Empire britannique et reflète le passage du mimétisme à l'hybridité dans le discours identitaire. Au Canada français, cela se traduit par des discussions sur la nécessité d'adopter le républicanisme et de se donner des emblèmes, comme un drapeau ou un hymne national, dénués de références britanniques, en plus de pousser les Canadiens français à embrasser une nouvelle identité, qu'elle soit continentale (américanité) ou territoriale (québécité). L'instauration de la république indienne devient une source d'inspiration pour certains intellectuels. Plusieurs éditorialistes proposent que le Canada français suive l'exemple de l'Inde dans sa quête d'une plus grande autonomie face à Londres. Certains vont même jusqu'à proposer l'indépendance comme en Inde. En ce sens, l'étude de la presse canadienne-française montre qu'il y a une réception positive face à l'indépendance indienne et que l'appropriation du discours de la décolonisation implique une mutation du discours nationaliste emprunté, en partie, à l'Inde. Cet emprunt accentue l'hybridité du discours identitaire canadien-français qui, désormais, se réfère aux conditions historiques des autres peuples, aussi lointains qu' ils puissent paraître. 


\section{La résistance à la guerre}

Héritiers d'une longue tradition de résistance à la guerre, plusieurs nationalistes canadiens-français ont souvent exprimé leur opposition aux guerres impériales qui avaient pour but la défense de l'Empire britannique; la guerre des Boers et la Grande Guerre en sont des exemples. Sous la direction d'André Laurendeau de 1937 à 1943, la revue L'Action nationale s'oppose farouchement à la guerre et à la conscription. Laurendeau écrit à plusieurs reprises dans L'Action nationale des textes sur Gandhi et sa lutte politique en Inde et en Afrique du Sud. Il consacre au Mahatma deux longs articles : le premier est à caractère biographique tandis que le second explique en quoi consiste la politique gandhienne de la non-coopération et de la non-violence, soit le satyagraha. Laurendeau affirme que l'action politique de Gandhi s'explique par le fait que

Londres avait promis aux Indiens de les traiter justement en Afrique-Sud si elle venait à bout des Boërs. Or après sa victoire, les Indiens furent plus durement harcelés qu’avant.

Surtout, en août 1917, le secrétaire d'État pour l'Inde, E. S. Montagu, avait promis à l'Inde un gouvernement responsable. Et « en aucun pays l'évangile wilsonien des Quatorze Points, le droit des peuples à disposer d'eux-mêmes, n'excitèrent pareille dévotion ». Cela donnera même lieu à une agitation dont Londres prit prétexte pour maintenir en temps de paix la dictature du temps de guerre ${ }^{13}$.

En présentant le satyagraha comme un outil de la non-coopération et de la désobéissance civile, Laurendeau fait le parallèle avec son propre mouvement contre la guerre et la conscription. Il affirme : « [S]i j'en avais eu l'héroïsme, ce qui m' eût attiré, ce n'est pas la guerre sacrée qu'on nous prêchait, mais la résistance passive de Gandhi ${ }^{14}$. "

${ }^{13}$ André Laurendeau, «Qui est Gandhi ? Gandhi et l'Empire britannique », L'Action nationale, avril 1943, p. 298-299.

${ }^{14}$ André Laurendeau, La crise de la conscription : 1942, Montréal, Éditions du Jour, 1962, p. 151. Gandhi s'inspirait d'Henry David Thoreau, un Américain d'origine 
S'avouant ébloui par Gandhi, Laurendeau concède : "J'ai voulu définir en quelques pages la naissance et la signification du gandhisme : assurément, c'était trop entreprendre. J'espère au moins n'avoir rien dit contre la vérité, - cette vérité qui, avec l'amour, est la seule passion du Mahatma ${ }^{15}$. » Il présente la lutte non violente de Gandhi comme un acte héroïque.

- Qu'est-ce que le Satyagraha? En voici une illustration bien superficielle.

- Le gouvernement prépare une loi que vous estimez injuste. Vous essayez de soulever l'opinion contre elle. Puis, vous prévenez le chef d'État que vous ne lui obéirez pas. Bien entendu, le gouvernement ne vous prend pas au sérieux et il vote sa loi. Vous y contrevenez consciemment et volontairement. Le gouvernement vous emprisonne : vous acceptez l'emprisonnement, même les travaux forcés, sans la moindre violence, sans le moindrement haïr votre persécuteur. Bien entendu, vous êtes par hypothèse un citoyen honnête, qui obéit généralement aux lois, qui a longuement pesé son acte, et qui désobéit par amour de la justice.

Votre peine une fois purgée, le gouvernement vous libère. Mais comme ni la solitude, ni les brimades, ni un régime alimentaire insuffisant, ni les ennuis subis par votre famille ne vous ont fait changer d'idée, vous n'avez rien de plus pressé que de violer la loi de nouveau ${ }^{16}$.

À cette position antimilitariste traditionnelle s'ajoute une dimension internationale puisque les anticonscriptionnistes canadiensfrançais situent leur refus de servir dans la mouvance des autres mouvements pacifistes dans le monde. Ainsi, Edmond Lemieux, de L'Action nationale, évoque plusieurs mouvements pacifistes internationaux pour ensuite établir un parallèle entre les opposants à la

française qui refusait de payer les taxes d'un gouvernement esclavagiste et en guerre contre le Mexique. Thoreau écrit La désobéissance civile en 1849.

${ }^{15}$ André Laurendeau, "Qui est Gandhi? Gandhi et l'Empire britannique », p. 301.

${ }^{16}$ Ibid., p. 292. 
conscription au Canada français et les mouvements pour la paix mondiale inspirés par Gandhi. L'auteur souligne que les mouvements pacifistes existent surtout depuis la fin de la Première Guerre (en réaction aux échecs des démocraties complices du carnage, à la montée du fascisme et au colonialisme, qui nie l'autodétermination des peuples), et que Gandhi en est l'un des principaux porte-paroles ${ }^{17}$.

Vers la fin des années 1930, devant la militarisation de l'Allemagne, la crainte d'un autre conflit mondial intensifie les déclarations pour la défense de l'Empire. Anatole Vanier, président de la Ligue d'action nationale, souligne que les membres du Congrès national indien de Nehru et de Gandhi ont voté « une résolution s'opposant à la participation de l'Inde aux guerres de l'Empire britannique. Au Canada que voyons-nous à côté et contre nos manifestations nationalistes, auxquelles, remarquons-le, les vieux partis qui se succèdent au pouvoir sont généralement étrangers ${ }^{18}$ ? " Vanier se plaint qu'aucun parti ne livre un message de paix comme Gandhi et que les roulements de tambours signifient que « [n] ous sommes en ce moment les témoins d'une profonde agitation impérialiste ${ }^{19}$ ". Georges Pelletier ajoute que de plus en plus d'associations et de clubs « ouvrent de temps à autre leur tribune à des tenants discrets de l'idée canadienne, servent au fond de véhicule ordinaire, de souple tremplin, si l'on peut dire, à la propagande impérialiste » et que " $[\mathrm{m}]$ ême des maharajahs de l'Inde servent de truchement à la propagande impériale $[\ldots]^{20}$ ».

La déclaration de guerre à l'Allemagne par l'Angleterre entraîne de facto l'Inde dans ce conflit. Tout comme la plupart des nationalistes canadiens-français, les leaders indiens, comme Nehru et Gandhi, refusent d'appuyer les efforts de guerre des alliés à moins que l'Inde participe en tant que démocratie indépendante. L'Action nationale

\footnotetext{
${ }^{17}$ Edmond Lemieux, "De la paix au pacifisme? ", L'Action nationale, vol. XXXIV, $\mathrm{n}^{\circ} 2$ (octobre 1949), p. 126-130.

${ }^{18}$ Anatole Vanier, «Politique extérieure », L’Action nationale, vol. IX, n 2 (février 1937), p. 94.

${ }^{19}$ Ibid., p. 95.

${ }^{20}$ Georges Pelletier, "La propagande impérialiste ", L'Action nationale, vol. XIII (juin 1939), p. 496-497.
} 
signale, dans sa rubrique intitulée "Calendrier de guerre ", que " [1]e Congrès pan-indien se réunit à Patna. Il se dissocie par résolution des "guerres impérialistes" de l'Angleterre. Il déclare : "La liberté hindoue ne saurait exister dans l'orbite de l'impérialisme anglais, et le status [sic] de dominion ou tout autre status [sic] dans les cadres de l'Empire ne sauraient s'appliquer à l'Inde" ${ }^{21}$."

Une fois la Deuxième Guerre mondiale bien entamée et qu'avec elle la conscription devienne possible, L'Action nationale crée un troisième parti (pour contrer les partis conscriptionnistes : le Parti libéral et le Parti conservateur). L'histoire retiendra que Laurendeau dirigea au Québec le Bloc populaire canadien, un parti politique de circonstance, qui se déclare contre la conscription. Denis Monière dira de Laurendeau qu' " en politique internationale, il aurait préféré voir le Canada suivre l'exemple de l'Inde et s'engager sur le chemin de la troisième voie, celle de la neutralité2 ${ }^{2}$ ». Laurendeau écrit :

De même, il faudrait réfléchir sur les peuples qui viennent de secouer leurs chaînes : l'Irlande, qui aboutit à De Valera; les Indes de Gandhi et de J. Nehru, le Portugal avec Salazar. Ces exemples ne sauraient nous fournir la réponse que nous cherchons, puisque chacun d'eux est tiré de situations concrètes bien différentes de la nôtre. Mais ils stimulent la pensée, et montrent comment ont abouti des mouvements que les réalistes avaient, bien entendu, étiquetés « impossibles $»^{23}$.

En se référant à l'Inde pour légitimer leur opposition à la conscription et à la guerre, les intellectuels du quotidien Le Devoir et de L'Action nationale trouvent un modèle international universellement perçu comme pacifiste. La personnalité de Gandhi fait l'unanimité sur le caractère pacifique de l'opposition à la guerre. Ce ralliement

${ }^{21}$ "Calendrier de guerre : à travers le Commonwealth", L'Action nationale, vol. XV, nº 6 (juin 1940), p. 474.

${ }^{22}$ Denis Monière, André Laurendeau et le destin d'un peuple, Montréal, Québec Amérique, 1983, p. 159.

${ }^{23}$ André Laurendeau, "L'impossible" troisième parti ", L'Action nationale, vol. XVII, n 4 (avril 1941), p. 273. 
au discours gandhien de la non-violence révèle, chez ces intellectuels, un esprit d'émulation face à une doctrine concoctée par d'autres, en l'occurrence des Indiens. Après la guerre, L'Action nationale publiera un long texte de Frank Emmanuel intitulé "Le pacifisme des forts ", dans lequel il présente Gandhi comme une personnalité exemplaire du pacifisme. "L'année 1948, qui vit mourir Gandhi, vient de voir aussi l'éveil, presque inapparent sous les Pouvoirs totalitaires, plus manifeste chez les nations dites démocratiques, d'une réaction populaire qui tend à renverser l'affreuse domination de la mort et de l'horreur ». L'auteur ajoute que « [c]'est à notre génération de fournir la réponse. C'est à elle de trouver une formule de doctrine et d'action qui permette à l'humanité de se dégager à la fois de la guerre impérialiste, des camps de concentration et de la famine ${ }^{24}$ ».

\section{L'indépendance de l'Inde et la décolonisation}

L'indépendance de l'Inde change en profondeur le vocabulaire nationaliste du monde entier. Le statut de colonisé est conceptualisé et adopté par les acteurs nationalistes qui, dans leur discours, mettent en lumière ce statut problématique en l'associant parfois à la violence. Victime de l'exploitation économique d'une puissance étrangère, le colonisé est aussi réprimé par l'État qui légitime sa condition d'exploité. Pour plusieurs colonisés, la fin du colonialisme se traduit par un passage obligé au socialisme, car le rôle de l'État postcolonial consiste à redistribuer la richesse et non plus à servir un système d'exploitation économique jugé violent par certains. Si l'État perpétue l'exploitation économique dans une ex-colonie (comme on l'a observé dans bon nombre de cas), il faut alors parler de néocolonialisme. Le nationalisme agit comme moteur des luttes anticoloniales. Pour de nombreux sympathisants canadiens-français, la lutte armée est à rejeter, mais la prise du pouvoir par un parti politique nationaliste décolonisateur est souhaitable. L'Action

${ }^{24}$ Frank Emmanuel, "Le pacifisme des forts ", L'Action nationale, vol. XXXIV, $\mathrm{n}^{\circ} 2$ (octobre 1949), p. 131, 148. 
nationale parle d'un nationalisme " d'émancipation ", celui des "nations opprimées qui revendiquent leur droit » en prenant Gandhi comme exemple.

Pour prendre un cas patent parmi ceux dont ils parlent, il serait plutôt difficile de prouver que le nationalisme de Gandhi consacre la souveraineté de la force; il est au contraire fondé sur un principe pacifiste de non-résistance par les armes, même aux attaques, qui est le contraire même de la loi de la force. Les nationalismes d'émancipation, dans la plupart des cas, consacrent et revendiquent, au contraire, la primauté des droits spirituels; [...] la plupart du temps alors informé par des mobiles de domination politique et économique, qui consacre le principe de la souveraineté de la force, grâce à laquelle des minorités économiques ou militaires ou même, comme au Canada, des majorités politiques établissent leur sujétion sur certains peuples, pour les exploiter politiquement et économiquement ${ }^{25}$.

Au début de la Révolution tranquille, Jacques Poisson, de L'Action nationale, mentionne dans un article intitulé «Fin du régime colonial » que trois partis ou regroupements incarnent ce nationalisme décolonisateur : l'Alliance laurentienne, l'Action socialiste pour l'indépendance du Québec et le Ralliement pour l'indépendance nationale (RIN) ${ }^{26}$. À ses yeux, ces regroupements s'inspirent d'un nationalisme décolonisateur porteur d'un message ambivalent qui oscille entre le désir de changement et le conservatisme.

L'Inde est aussi le chef du mouvement non-aligné. Jugée utopiste par certains, sa politique extérieure reflète une quête d'identité gandhienne qui rejette la guerre. De plus, elle montre aux nations colonisées qu'il est possible de s'affranchir des superpuissances en temps de guerre froide. De par son caractère démocratique, l'Inde se pose comme porte-parole crédible des peuples opprimés. Ses prises

\footnotetext{
25 "Entre deux mots", L'Action nationale, vol. XXV, n 6 (juin 1945), p. 485.

${ }^{26}$ Jacques Poisson, "Fin du régime colonial ", L'Action nationale, vol. L, n ${ }^{\circ} 6$ (février 1961), p. 508-522. Voir plus spécifiquement la section «Le Québec et la décolonisation ».
} 
de position sur la scène internationale soulèvent des commentaires élogieux dans la presse nationaliste canadienne-française, qui évoque le réveil normal de l'Asie et des peuples opprimés. Roger Duhamel écrit que l'Inde partage l'idéal démocratique implanté par la révolution américaine et que son indépendance peut servir d'exemple aux autres peuples de la terre.

On peut parfois moquer doucement les naïvetés de l'idéalisme américain. Sans doute. Ne faut-il pas toutefois admettre qu'il demeure un beau témoignage en faveur de l'humanité? Quand nous risquons, au spectacle de l'égoïsme, de perdre cœur, il fait bon de penser que notre grande nation voisine, qui commet elle aussi des fautes, ne perd jamais cependant le sentiment de la justice et recherche son royaume sur terre. À cet égard, La révolution de l'Inde a une valeur de symbole [...] on découvre le souci de remettre dans le monde un peu plus d'harmonie, un peu plus de bien-être, un peu plus d'équité. Et c'est là une chose très belle ${ }^{27}$.

De tous les journaux canadiens-français, Le Devoir offre de loin la plus grande couverture à l'indépendance de l'Inde. Le penchant nationaliste de ce journal est bien connu, et ses nombreux éditoriaux sur l'Inde montrent clairement sa sympathie envers son indépendance, et ce, tout au long de l'entre-deux-guerres. Paul Sauriol, Omer Héroux et Pierre Vigeant signent plusieurs éditoriaux plus que sympathiques à la cause, appelant les Canadiens français à suivre l'exemple de l'Inde dans la lutte nationaliste visant à réduire les liens avec l'Empire britannique. Dans un article tranchant, Sauriol souligne que l'Inde se bat contre l'impérialisme et que sa lutte inspire les peuples de la terre. Il cite les paroles de Nehru qui déclare que la lutte pour l'indépendance de l'Inde est « [...] une partie de la lutte de tous les peuples opprimés du monde, et nous ne perdrons jamais de vue ce fait ${ }^{28}$ ". Sauriol rappelle que la Charte de l'Atlantique a promis à tous les

${ }^{27}$ Roger Duhamel, «Courrier des lettres : la révolution de l'Inde ", L'Action nationale, vol. XXV, no 4 (avril 1945), p. 305-306. En italique dans le texte.

${ }^{28}$ Paul Sauriol, "Aux Indes : la lutte d'un pays contre l'impérialisme ", Le Devoir, 18 juillet 1945 . 
peuples la liberté de disposer d'eux-mêmes, et ce, malgré les tensions communales en Inde : "Si les grandes puissances manifestaient plus de sincérité dans leurs relations internationales, elles seraient mieux placées pour convaincre leurs coloniaux qu'ils ont encore besoin de conseils; mais ce qui se passe en Europe n'est guère édifiant ${ }^{29}$. "Sauriol invite les Canadiens français à " chercher en Asie des leçons d'indépendance $^{30}$ ". Son collègue Omer Héroux considère, pour sa part, les indépendances indienne et birmane comme un "beau sujet de méditation pour les Canadiens trop timides».

Devant cette affirmation du chef du gouvernement anglais que celui-ci n'entend garder personne malgré soi dans l'Empire ou le Commonwealth, devant cet aveu du protagoniste par excellence de l'impérialisme que l'indépendance est la destinée naturelle d'un pays comme la Birmanie, les Canadiens trop timides s'aviseront-ils enfin de regarder en face la destinée logique de leur pays : l'indépendance totale, et de faire la seule politique qui lui convient, celle qui s'inspire d'abord des intérêts du Canada ${ }^{31}$.

Pierre Vigeant perçoit l'indépendance de l'Inde comme un tournant majeur dans l'histoire internationale. Il écrit que « [1]'émancipation politique de l'Inde est interprété [sic] un peu partout comme la fin de l'ère d'impérialisme qui a atteint son apogée au XIX ${ }^{\mathrm{e}}$ siècle $^{32}$ ". Avec l'indépendance de l'Inde commence l'histoire de la décolonisation, un processus qui prendra une trentaine d'années avant d'être achevé. D'autres journaux, notamment La Tribune, Le Canada et L'Action catholique se montrent favorables à l'indépendance indienne. Ils affirment que cet événement marque un changement fondamental

${ }^{29}$ Paul Sauriol, "Les traités balkaniques seront-ils étudiés à trois ou à cinq? ", Le Devoir, 28 septembre 1945.

${ }^{30}$ Paul Sauriol, "La Birmanie en marche vers l'indépendance ", Le Devoir, 29 janvier 1947.

${ }^{31}$ Omer Héroux, « Nous ne souhaitons retenir personne contre son gré dans l'Empire et le Commonwealth : beau sujet de méditation pour les Canadiens trop timides ", Le Devoir, 23 décembre 1946.

32 Pierre Vigeant, "Les puissances coloniales européennes contraintes de se retirer de l'Asie ", Le Devoir, 15 août 1947. 
des mentalités et de l'histoire humaine. La Tribune mentionne que " c'est là un évènement historique de la plus haute importance ${ }^{33}$ " et Le Canada signale que

[la] date du 15 août mérite de passer à l'histoire, au même titre que les dates universellement célèbres du 4 juillet, jour de l'indépendance américaine, du 14 juillet, anniversaire de la prise de la Bastille et fête de la République française. [...] le changement de mode de gouvernement a été obtenu et s'est accompli par des moyens pacifiques. Le fait est sans précédent et il ne fait aucun doute que l'histoire en gardera le souvenir ${ }^{34}$.

L'Action catholique ajoute que «[c]ette évolution des Indes vers l'indépendance est une des conséquences de la guerre, une application vraiment pratique des principes pour lesquels les Nations-Unies [sic] se sont battues ${ }^{35}$ ». Ainsi, l'autodétermination des peuples imaginée par Wilson devient possible après la Deuxième Guerre. Au même titre qu'une Charte de l'Atlantique, une Charte de l'Est est proposée, incluant l'Inde, chef de file de ce nouveau mouvement d'émancipation. François-Albert Angers, de L'Action nationale, écrit qu'il est " nécessaire de signaler que les Canadiens français ont tout intérêt à s'en faire les propagandistes au Canada ainsi qu' à appuyer les revendications pour une Inde libérée ${ }^{36}$ ». En publiant un long texte d'un Anglo-Canadien favorable à l'autonomie de l'Inde, L'Action nationale explique qu' " [a]utonomistes nous-mêmes, et sans aucun de ces préjugés de race ou de couleur qui obnubilent l'esprit de certains dirigeants d'Empire, nous avons toujours manifesté la plus extrême sympathie pour la cause indienne ${ }^{37}$ ». Sous la plume de Léopold

\footnotetext{
33 "L'indépendance de l'Inde ", La Tribune, 15 août 1947.

${ }^{34}$ Guy Jasmin, «Deux nouveaux Dominions », Le Canada, 16 août 1947.

${ }^{35}$ Louis-Philippe Roy, " 300 millions d'hommes émancipés ", L'Action catholique, 14 août 1947 .

${ }^{36}$ François-Albert Angers, "Une lueur d'espoir! ", L’Action nationale, vol. XIX, no 3 (avril 1942), p. 233.

${ }^{37}$ Edmond Lemieux, "Témoignage anglo-canadien sur la crise indienne », L'Action nationale, vol. XX, no 1 (septembre 1942), p. 76.
} 
Richer, l'hebdomadaire Notre Temps affirme que l'Inde est « le centre d'attraction naturel d'une fédération asiatique dont il est déjà possible d'entrevoir l'influence politique et culturelle ». Rendant hommage au leadership de Nehru dans le réveil de l'Asie, ce journal affirme :

Le désir d'émancipation et d'indépendance gît au fond du cœur de tous les hommes et de toutes les nations. Par des chemins divers et malgré des obstacles formidables, les peuples concentrent leurs forces vers la création d'une fédération asiatique. C'est dans cette partie du monde que se dérouleront les événements capitaux de la fin du $\mathrm{Xx}^{\mathrm{e}}$ siècle $^{38}$.

L'indépendance de l'Inde trouve aussi ses détracteurs dans la presse canadienne-française. Le journal La Presse publie des reportages nettement en faveur du maintien du lien colonial et souligne le danger que représente la partition. Alfred Ayotte, entre autres, maintient que le projet d'indépendance est insoutenable, car il implique une partition impraticable ${ }^{39}$. La Presse indique que les violences communales en Inde « inclinent naturellement les étrangers à se demander si l'Inde est bien mûre pour l'autonomie, pour la liberté qu'elle sollicite $^{40}$ ». La Presse fait preuve de beaucoup d'optimisme quant aux chances d'éviter la partition. Évoquant la dualité culturelle du Canada, ce journal publie davantage les reportages vantant les mérites des unions pluriculturelles et multiplie les points de vue des Indiens qui favorisent l'unité au détriment de la partition.

Ce besoin d'unité, a-t-il dit (Dr. D. P. Pandia), si nécessaire au progrès et au développement du commerce, de l'industrie, du transport, des finances, des douanes, de la défense et des

${ }^{38}$ Pierre George, "Vers un bloc asiatique ", Notre Temps, 16 août 1947.

${ }^{39}$ Alfred Ayotte, «L'Inde, État démocratique », La Presse, 14 mai 1946. L'Inde et le Pakistan naissent dans la partition. La partie est du Pakistan deviendra le Bangladesh en 1971. L'Inde fut constituée à partir des grandes provinces sous administration britannique, en plus des États princiers qui composent le sous-continent indien. L'État princier du Cachemire demeure divisé aujourd'hui et constitue une source de méfiance mutuelle entre le Pakistan et l'Inde.

40 "Est-on prêt pour l'autonomie? ", La Presse, 20 septembre 1946. 
communications, poussera les deux États indiens du Pakistan et de l'Hindoustan à se fédérer en une seule nation comme ce fut le cas pour le Canada en $1867^{41}$.

Au moment de l'indépendance, La Presse et Le Soleil publient des articles beaucoup plus élogieux envers l'Angleterre et sa " générosité " à l'égard de l'Inde. La Presse ira même jusqu'à célébrer la décision de l'Inde de demeurer dans le Commonwealth, car « il semble qu'il lui sera avantageux de conserver cette union, à une époque où le sort des petites nations isolées est exposé à bien des dangers ${ }^{42}$ ». Il est, par ailleurs, assez étonnant que l'on puisse qualifier l'Inde de petite nation alors qu'elle représente un sous-continent. À Québec, Le Soleil sous-estime le mouvement de décolonisation mis en branle par Nehru et aborde l'indépendance indienne non pas comme une victoire historique des colonisés sur le pouvoir impérial, mais plutôt comme la preuve de l'efficience du Parlement britannique dont le Québec est encore tributaire.

Sur ce point, il ne saurait y avoir qu'un jugement favorable à l'honneur anglais [...] Ainsi donc, la Grande-Bretagne est la première puissance impériale à remplir une obligation prise publiquement, en rendant leur indépendance politique aux peuples qu'elle a entraînés dans l'art du gouvernement. Il faudra probablement attendre longtemps avant que son exemple soit suivi par d'autres nations coloniales ${ }^{43}$.

Outre les clivages de la presse canadienne-française au sujet de l'indépendance de l'Inde, l'événement marque pour tous les peuples le début de l'ère postcoloniale. Le Canada français entame sa réflexion sur son propre postcolonialisme, sur les symboles de l'Empire qui sont à rejeter et sur ceux qu'il faut construire. Tout comme l'Inde, le Canada français se donne des symboles nationaux en adoptant un

\footnotetext{
${ }^{41}$ Bureau United Press, «Les Indiens bientôt unis : entrevue avec le Dr. D. P. Pandia ", La Presse, 6 août 1947.

42 "L'Inde autonome », La Presse, 15 août 1947.

43 "L’indépendance à tout prix ", Le Soleil, 15 août 1947.
} 
drapeau et un hymne national. Ces symboles confirment la fin du mimétisme dans le discours national, qui emprunte désormais une forme plus hybride faisant référence aux autres peuples de la terre. Les parallèles qu'établissent les intellectuels canadiens-français entre les symboles de l'indépendance adoptés par l'Inde et ceux adoptés par le Canada français montrent un changement dans la problématique identitaire. Dorénavant, l'autonomie canadienne-française trouve dans celle de l'Inde un lien de parenté constitutionnelle.

\section{Symbolisme}

Bien qu'il ne s'agisse en fait que de tissu, les drapeaux jouent un rôle identitaire fondamental. Tout comme l'hymne national ou les armoiries, les symboles des pays génèrent une identité et une fierté consensuelles. Comme le soulignent Marie-France Wagner et Lyse Roy, l'organisation d'une société donnée est fondée sur un certain nombre de systèmes symboliques qui se structurent en fonction du temps et d'espaces spécifiques ${ }^{44}$. Le God Save the Queen et l'Union Jack (ou le Red-Ensign) furent rejetés massivement par les Canadiens français, et les résultats des référendums sur la conscription attestent leur refus de défendre l'Empire britannique et ses symboles. Pour ce qui est des symboles autochtones, les Canadiens français en ont longtemps arboré des officieux. L'Ô Canada et le Sacré-Cour servaient déjà bien avant l'indépendance de l'Inde, mais ne furent jamais adoptés officiellement.

Le comité Euvre du drapeau national et le curé Elphège Filiatrault publient plusieurs brochures en faveur de l'adoption d'un drapeau national au début du $\mathrm{Xx}^{\mathrm{e}}$ siècle ${ }^{45}$. Avec un certain succès, le fleurdelisé sera adopté par l'ensemble du Canada français, mais du côté du Canada anglais, l'Union Jack demeure. Dans le but de faire renaître le projet d'un drapeau national, la Ligue de la Propagande

\footnotetext{
${ }^{44}$ Marie-France Wagner et Lyse Roy, "Rituels et cérémonies du Xvi ${ }^{\mathrm{e}}$ au XXI ${ }^{\mathrm{e}}$ siècle ", Bulletin d'histoire politique, vol. 14, $\mathrm{n}^{\circ} 1$ (automne 2005), p. 7.

${ }^{45}$ Elphège Filiatrault, Aux Canadiens français : notre Drapeau, St-Hyacinthe, Imprimerie La Tribune, 1903.
} 
du drapeau national des Canadiens français, associée à L'Action nationale, entame une campagne politique au milieu des années 1930. La guerre et la conscription repoussent à plus tard l'adoption d'un drapeau, sujet de divisions additionnelles, mais une fois la guerre terminée, un comité parlementaire du gouvernement canadien est mis sur pied pour créer un drapeau canadien. Devant cet état de fait, l'Assemblée législative du Québec demandait au fédéral de choisir un drapeau qui « exclut tout signe de servage, de colonialisme et que peut arborer fièrement tout Canadien sans distinction d'origine ${ }^{46} »$.

Au lendemain de la Deuxième Guerre mondiale, l'Angleterre perd considérablement de sa puissance d'antan, laissant aux colonies le choix de demeurer dans le Commonwealth ou non. Au Canada français, plusieurs nationalistes proposent l'établissement d'une république afin de couper les liens monarchiques avec l'Angleterre, et font campagne pour l'adoption du fleurdelisé comme drapeau national. Citant en exemple l'établissement d'une république en Inde (qui demeure toutefois au sein du Commonwealth), L'Action nationale évoque ce précédent pour promouvoir la possibilité de couper les liens avec la monarchie et ses symboles. Jacques Perrault, avocat gauchiste qui a combattu Duplessis, prédit que l'indépendance de l'Inde accentuera le républicanisme canadien.

C'est ainsi que l'Inde s'est constituée en république en demeurant, pour le moment du moins, dans le "Commonwealth " à titre de Dominion.

Le jour où le Canada élira un parlement avec le mandat de transformer en république notre pays, il suffira d'un vote des deux Chambres du parlement pour que le parlement de GrandeBretagne, lié par les précédents indiqués plus haut, acquiesce à ce changement constitutionnel fondamental. [...]

[...] conséquence prévisible dès maintenant, la république canadienne, se joignant au système pan-américain, rompra tout lien

${ }^{46}$ Luc Bouvier, "Vers le fleurdelisé ", L'Action nationale, vol. LXXXVI, n ${ }^{\circ} 9$ (novembre 1996), p. 102. 
avec le "Commonwealth " britannique. Quelles obligations la nouvelle république assumera-t-elle, en particulier vis-à-vis son puissant voisin, les États-Unis?

Ces difficultés - quelle évolution en ce monde en est exempte?ne sauraient effrayer un Canada audacieux, voulant cesser, enfin, d'être à la remorque de l'Angleterre et devenir vraiment un État libre et souverain ${ }^{47}$.

Pierre Vigeant, du journal Le Devoir, croit, pour sa part, que l'exemple de l'Inde devrait inciter le Canada à sortir du Commonwealth ou, du moins, à couper les liens avec la monarchie anglaise.

Si le statut de Dominion ne semblait guère alléchant pour les Indiens, est-ce que les Canadiens doivent s'en contenter? Si les Indiens réservent leur droit de sortir du Commonwealth et le font reconnaître par la Grande-Bretagne, les Canadiens qui constituent un État d'Amérique ne doivent-ils pas en envisager l'éventualité? Il n'est pas sûr qu'ils ne devancent pas le Canada dans la voie de l'indépendance, qu'ils rejettent avant nous l'allégeance à un roi étranger ${ }^{48}$.

Les campagnes en faveur de l'adoption d'un drapeau furent souvent associées aux mouvements contre la guerre et la conscription. La campagne du début du $\mathrm{Xx}^{\mathrm{e}}$ siècle est liée à la guerre des Boers tandis que celle qui nous intéresse ici se déroule juste après la Deuxième Guerre. Anatole Vanier, de L'Action nationale, dirigera la Ligue d'action nationale mandatée pour canaliser la pression populaire en vue de l'adoption d'un drapeau. L'Action nationale rappelle qu' « [u] n drapeau, ce n'est qu'un symbole, mais c'est un symbole nécessaire qui aide un peuple à prendre conscience de lui-même. Il évoque son passé, lui fixe un idéal de vie collective et l'invite au ralliement ${ }^{49}$ ". De son côté, l'Inde ne tarde pas à remplacer les symboles britanniques par son

${ }^{47}$ Jacques Perrault, «La "Couronne” et la Constitution canadienne », L'Action nationale, vol. XXXII, n 2 (octobre 1948), p. 123, 125.

48 Pierre Vigeant, "Dominion ou république », Le Devoir, 4 juin 1947.

${ }^{49}$ René Chaloult, "Un drapeau provincial ", L'Action nationale, vol. XXXI, $n^{\circ} 1$ (janvier 1948), p. 5. 
propre drapeau (juillet 1947), ses armoiries et son hymne national (janvier 1950). Ces faits ne passent pas inaperçus chez les nationalistes canadiens-français. Le journal Le Canada cite Nehru lorsqu’il présente le nouveau drapeau indien : ce " n'est pas un drapeau d'impérialisme, un drapeau de dominion sur qui que ce soit, mais un drapeau de liberté, non seulement pour nous, mais pour tous les peuples qui le verront $[\ldots]^{50}$ ". Au moment de l'adoption de ce drapeau, La Patrie affirme que c'est un " [b]eau jour pour l'Inde ». Le journal cite les propos de Nehru, qui proclame un jour de réjouissance pour le monde entier $^{51}$. De son côté, Le Devoir publie un dépêche de l'American Press intitulée "L'Inde se choisit un drapeau distinctif ", en soulignant qu'il " ne contient aucun signe qui pourrait marquer le lien avec l'Empire britannique ${ }^{52}$ ». Le Devoir ajoute en sous-titre «À quand le drapeau véritablement canadien?".

La situation diffère singulièrement du Canada. Notre pays a obtenu son indépendance en 1931, par le statut de Westminster, et nous n'avons pas encore de drapeau distinctif. Le premier ministre King n'a pas jugé bon de donner suite aux demandes qui lui ont été faites de faire adopter par le Parlement un projet de drapeau distinctif [...] Il faut croire qu'il coule moins de sang britannique dans les veines des députés hindous que dans les veines de certains députés canadiens. Là-bas, l'on n'a pas attendu 15 ans avant d'adopter un drapeau vraiment distinctif. À quand le drapeau véritablement canadien ${ }^{53}$ ?

Dans un ultime article de janvier 1948, mois durant lequel l'Assemblée législative du Québec adopte finalement le fleurdelisé, L'Action nationale presse le gouvernement québécois d'adopter un drapeau, tout comme vient de le faire l'Inde.

Le premier geste de l'Inde, après avoir conquis son indépendance, fut de déployer au vent son emblème. Le 15 août dernier, devant

\footnotetext{
${ }^{50}$ Guy Jasmin, "Deux nouveaux Dominions », Le Canada, 16 août 1947.

51 " Beau jour pour les Indes ", La Patrie, 17 août 1947.

${ }^{52}$ Le Devoir, 22 juillet 1947.

${ }^{53}$ Ibid.
} 
le parlement des Nations-Désunies, à Lake Success, on descendit le drapeau aux couleurs britanniques pour hisser solennellement le nouveau drapeau national. [...] Ainsi, à Québec, ne faut-il pas espérer que le remplacement de l'Union Jack par le Fleurdelisé estomperait un peu le pénible souvenir de nos trop légitimes griefs?... [...] À Québec, ce n'est pas même le Red Ensign qui flotte sur les édifices du gouvernement, mais un drapeau complètement étranger : L'Union Jack, [...] Voulons-nous, comme l'Inde indépendante, comme l'État libre d'Irlande, comme la nouvelle Acadie remplacer cet emblème étranger par le nôtre ${ }^{54}$ ?

Malgré l'adoption du fleurdelisé par le Parlement québécois, le Canada hésitera encore pendant près de vingt ans avant d'adopter son propre drapeau. Dans l'impatience de voir le Canada rompre ses liens avec l'Angleterre, Antonio Perrault, de L'Action nationale, lance une boutade au Parlement canadien.

Il faudra, en somme, que le Canada suive - sujet à modifications la procédure qui permit à l'Irlande du Sud et à l'Inde d'être reconnues comme républiques.

Je vous entends : dans ce cas, ce n'est pas pour maintenant. Imaginez-vous un Louis Saint-Laurent présentant aux Communes une telle législation? S'il avait à ce sujet le courage et la fierté d'un Valera et d'un Nehrou [sic], quelle crise de nerfs chez Drew, Diefenbaker, Coldwell, Low, les rédacteurs du Star et de la Gazette, tous plus britanniques et impérialistes que Canadiens ${ }^{55}$.

\section{Conclusion}

L'étude des références à l'Inde dans le discours nationaliste canadienfrançais ouvre une piste de réflexion sur la connectivité des colonies au sein de l'Empire britannique. S'étant mutuellement influencées dans la construction de leur discours politique et de leur identité

\footnotetext{
${ }^{54}$ Chaloult, "Un drapeau provincial », p. 6, 7, 8.

55 Antonio Perrault, "L'indépendance du Canada ", L'Action nationale, vol. XXXV, $\mathrm{n}^{\circ} 6$ (juin 1950), p. 429.
} 
postcoloniale, les voix des peuples subalternes ont façonné l'histoire politique. Ce survol de la presse canadienne-française concernant la lutte que l'Inde a menée pour obtenir sa libération indique un clivage politique entre le statu quo constitutionnel de l'Empire et le romantisme politique que génèrent les indépendances nationales. Des journaux moins nationalistes comme Le Soleil et La Presse favorisent davantage le maintien du lien britannique. Bien qu'ils ne s'opposent pas à l'indépendance de l'Inde, ils soulèvent des doutes sur la capacité de ses élites à maintenir l'unité du pays. L'indépendance indienne aura suscité un vif intérêt surtout chez les Canadiens français, lecteurs de journaux plus nationalistes comme Le Devoir et la revue L'Action nationale. Les observations que ces périodiques ont publiées sur l'Inde ont fait apparaître les concepts liés à la décolonisation dans le discours nationaliste au Canada français bien avant la Révolution tranquille, tout en laissant entendre que le nationalisme de Gandhi et de Nehru s'inscrivait dans une lutte politique légitime à laquelle se sont identifiés certains Canadiens français. Leurs commentaires généralement positifs envers l'indépendance indienne donnent raison au haut-commissaire de l'Inde au Canada, qui rapportait qu' elle était perçue comme un symbole de liberté.

La naissance de l'Inde a rendu possible l'autodétermination des peuples telle que prescrite par Woodrow Wilson. Aux yeux de certains nationalistes canadiens-français, l'indépendance de l'Inde signifie la fin de l'Empire britannique et enclenche le processus qui a mené à la transformation du nationalisme canadien-français. L'Action nationale a joué un rôle important dans la diffusion du vocabulaire et des concepts liés à la décolonisation. Les auteurs de la littérature de la décolonisation ont puisé leurs connaissances non seulement dans les œuvres de Memmi et de Fanon, mais aussi dans les revues et journaux québécois, qui se sont faits l'écho de la transformation internationale amorcée, entre autres, par la décolonisation de l'Inde. 